\title{
Dynamics of a discrete Lotka-Volterra model
}

Qamar Din

"Correspondence: qamar.sms@gmail.com

University of Azad Jammu and Kasmir, Muzaffarabad, Pakistan
(C) 2013 Din; licensee Springer. This is an Open Access article distributed under the terms of the Creative Commons Attribution License (http://creativecommons.org/licenses/by/2.0), which permits unrestricted use, distribution, and reproduction in any medium, provided the original work is properly cited.

\section{Abstract}

In this paper, we study the equilibrium points, local asymptotic stability of equilibrium points, and global behavior of equilibrium points of a discrete Lotka-Volterra model given by

$$
x_{n+1}=\frac{\alpha x_{n}-\beta x_{n} y_{n}}{1+\gamma x_{n}}, \quad y_{n+1}=\frac{\delta y_{n}+\epsilon x_{n} y_{n}}{1+\eta y_{n}}
$$

where parameters $\alpha, \beta, \gamma, \delta, \epsilon, \eta \in \mathbb{R}^{+}$, and initial conditions $x_{0}, y_{0}$ are positive real numbers. Moreover, the rate of convergence of a solution that converges to the unique positive equilibrium point is discussed. Some numerical examples are given to verify our theoretical results.

MSC: 39A10; 40A05

Keywords: difference equations; equilibrium points; local stability; global character

\section{Introduction and preliminaries}

Many authors investigated the ecological competition systems governed by differential equations of Lotka-Volterra type. Many interesting results related with the global character and local asymptotic stability have been obtained. We refer to $[1,2]$ and the references therein. Already, many authors [3, 4] have argued that the discrete time models governed by difference equations are more appropriate than the continuous ones when the populations are of non-overlapping generations. Particularly, the persistence, boundedness, local asymptotic stability, global character, and the existence of positive periodic solutions.

The discrete Lotka-Volterra models have many applications in applied sciences. Such models were first established in mathematical biology, and then their applications were spread to other fields [5-8]. Several variations of the Lotka-Volterra predator-prey model have been proposed that offer more realistic descriptions of the interactions of the populations. If the population of rabbits is always much larger than the number of foxes, then the considerations that entered into the development of the logistic equation may come into play. If the number of rabbits becomes sufficiently great, then the rabbits may be interfering with each other in their quest for food and space. One way to describe this effect mathematically is to replace the original model by the more complicated system. Most predators feed on more than one type of food. If the foxes can survive on an alternative resource, although the presence of their natural prey (rabbits) favors growth, a possible alternative model is the discrete dynamical system

$$
x_{n+1}=\frac{\alpha x_{n}-\beta x_{n} y_{n}}{1+\gamma x_{n}}, \quad y_{n+1}=\frac{\delta y_{n}+\epsilon x_{n} y_{n}}{1+\eta y_{n}},
$$


where parameters $\alpha, \beta, \gamma, \delta, \epsilon, \eta \in \mathbb{R}^{+}$, and initial conditions $x_{0}, y_{0}$ are positive real numbers.

It is a well-known fact that the discrete-time type models described by difference equations are more suitable than the continuous-time models. Nonlinear difference equations of order greater than one are of paramount importance in applications. Such equations also appear naturally as discrete analogues and as numerical solutions of differential and delay differential equations which model various diverse phenomena in biology, ecology, physiology, physics, engineering, and economics. Rational difference equations are a special form of nonlinear difference equations. We refer to [9-14] for basic theory of difference equations and rational difference equations. Recently, many authors have discussed the dynamics of rational difference equations [15-27].

\section{Linearized stability}

Let us consider a two-dimensional discrete dynamical system of the form

$$
\begin{aligned}
& x_{n+1}=f\left(x_{n}, y_{n}\right), \\
& y_{n+1}=g\left(x_{n}, y_{n}\right), \quad n=0,1, \ldots,
\end{aligned}
$$

where $f: I \times J \rightarrow I$ and $g: I \times J \rightarrow J$ are continuously differentiable functions and $I, J$ are some intervals of real numbers. Furthermore, a solution $\left\{\left(x_{n}, y_{n}\right)\right\}_{n=0}^{\infty}$ of the system (2.1) is uniquely determined by initial conditions $\left(x_{0}, y_{0}\right) \in I \times J$. An equilibrium point of $(2.1)$ is a point $(\bar{x}, \bar{y})$ that satisfies

$$
\begin{aligned}
& \bar{x}=f(\bar{x}, \bar{y}), \\
& \bar{y}=g(\bar{x}, \bar{y}) .
\end{aligned}
$$

Definition 2.1 Let $(\bar{x}, \bar{y})$ be an equilibrium point of the system (2.1).

(i) An equilibrium point $(\bar{x}, \bar{y})$ is said to be stable if for every $\varepsilon>0$ there exists $\delta>0$ such that for every initial condition $\left(x_{0}, y_{0}\right)$ if $\left\|\left(x_{0}, y_{0}\right)-(\bar{x}, \bar{y})\right\|<\delta$ implies $\left\|\left(x_{n}, y_{n}\right)-(\bar{x}, \bar{y})\right\|<\varepsilon$ for all $n>0$, where $\|\cdot\|$ is the usual Euclidean norm in $\mathbb{R}^{2}$.

(ii) An equilibrium point $(\bar{x}, \bar{y})$ is said to be unstable if it is not stable.

(iii) An equilibrium point $(\bar{x}, \bar{y})$ is said to be asymptotically stable if there exists $\eta>0$ such that $\left\|\left(x_{0}, y_{0}\right)-(\bar{x}, \bar{y})\right\|<\eta$ and $\left(x_{n}, y_{n}\right) \rightarrow(\bar{x}, \bar{y})$ as $n \rightarrow \infty$.

(iv) An equilibrium point $(\bar{x}, \bar{y})$ is called a global attractor if $\left(x_{n}, y_{n}\right) \rightarrow(\bar{x}, \bar{y})$ as $n \rightarrow \infty$.

(v) An equilibrium point $(\bar{x}, \bar{y})$ is called an asymptotic global attractor if it is a global attractor and stable.

Definition 2.2 Let $(\bar{x}, \bar{y})$ be an equilibrium point of a map $F(x, y)=(f(x, y), g(x, y))$, where $f$ and $g$ are continuously differentiable functions at $(\bar{x}, \bar{y})$. The linearized system of (2.1) about the equilibrium point $(\bar{x}, \bar{y})$ is given by

$$
X_{n+1}=F\left(X_{n}\right)=F_{J} X_{n},
$$

where $X_{n}=\left(\begin{array}{l}x_{n} \\ y_{n}\end{array}\right)$ and $F_{J}$ is a Jacobian matrix of the system (2.1) about the equilibrium point $(\bar{x}, \bar{y})$. 
Let $(\bar{x}, \bar{y})$ be an equilibrium point of the system (1.1), then

$$
\bar{x}=\frac{\alpha \bar{x}-\beta \bar{x} \bar{y}}{1+\gamma \bar{x}}, \quad \bar{y}=\frac{\delta \bar{y}+\epsilon \bar{x} \bar{y}}{1+\eta \bar{y}} .
$$

Hence, $O=(0,0), P=\left(\frac{\beta-\beta \delta+(-1+\alpha) \eta}{\beta \epsilon+\gamma \eta}, \frac{\gamma(-1+\delta)+(-1+\alpha) \epsilon}{\beta \epsilon+\gamma \eta}\right), Q=\left(\frac{-1+\alpha}{\gamma}, 0\right)$, and $R=\left(0, \frac{-1+\delta}{\eta}\right)$ are equilibrium points of the system (1.1). Then, clearly, $P=\left(\frac{\beta-\beta \delta+(-1+\alpha) \eta}{\beta \epsilon+\gamma \eta}, \frac{\gamma(-1+\delta)+(-1+\alpha) \epsilon}{\beta \epsilon+\gamma \eta}\right)$ is the unique positive equilibrium point of the system (1.1), if $\alpha>1, \delta \leq 1, \epsilon>\frac{\gamma-\gamma \delta}{\alpha-1}$ or $\alpha>1$, $\delta>1, \eta>\frac{-\beta+\beta \delta}{-1+\alpha}$.

The Jacobian matrix of the linearized system of (1.1) about the fixed point $(\bar{x}, \bar{y})$ is given by

$$
F_{J}(\bar{x}, \bar{y})=\left[\begin{array}{cc}
\frac{\alpha-\bar{y} \beta}{(1+\bar{x} \gamma)^{2}} & -\frac{\bar{x} \beta}{1+\bar{x} \gamma} \\
\frac{\bar{y} \epsilon}{1+\bar{y} \eta} & \frac{\delta+\bar{x} \epsilon}{(1+\bar{y} \eta)^{2}}
\end{array}\right] .
$$

Theorem 2.3 For the system $X_{n+1}=F\left(X_{n}\right), n=0,1, \ldots$, of difference equations such that $\bar{X}$ is a fixed point of $F$. If all eigenvalues of the Jacobian matrix $J_{F}$ about $\bar{X}$ lie inside the open unit disk $|\lambda|<1$, then $\bar{X}$ is locally asymptotically stable. If one of them has a modulus greater than one, then $\bar{X}$ is unstable.

\section{Main results}

Theorem 3.1 Assume that $\alpha<1$ and $\delta<1$, then the following statements are true.

(i) The equilibrium point $O=(0,0)$ is locally asymptotically stable.

(ii) The equilibrium point $Q=\left(\frac{-1+\alpha}{\gamma}, 0\right)$ is unstable.

(iii) The equilibrium point $R=\left(0, \frac{-1+\delta}{\eta}\right)$ is unstable.

Proof (i) The Jacobian matrix of the linearized system of (1.1) about the fixed point $(0,0)$ is given by

$$
F_{J}(0,0)=\left[\begin{array}{ll}
\alpha & 0 \\
0 & \delta
\end{array}\right] .
$$

Moreover, the eigenvalues of the Jacobian matrix $J_{F}(0,0)$ about $(0,0)$ are $\lambda_{1}=\alpha<1$ and $\lambda_{2}=\delta<1$. Hence, the equilibrium point $(0,0)$ is locally asymptotically stable.

(ii) The Jacobian matrix of the linearized system of (1.1) about the fixed point $\left(\frac{-1+\alpha}{\gamma}, 0\right)$ is given by

$$
F_{J}\left(\frac{-1+\alpha}{\gamma}, 0\right)=\left[\begin{array}{cc}
\frac{1}{\alpha} & \frac{\beta-\alpha \beta}{\alpha \gamma} \\
0 & \frac{(\alpha-1) \epsilon+\delta \gamma}{\gamma}
\end{array}\right] .
$$

The eigenvalues of the Jacobian matrix $J_{F}\left(\frac{-1+\alpha}{\gamma}, 0\right)$ about $\left(\frac{-1+\alpha}{\gamma}, 0\right)$ are $\lambda_{1}=\frac{1}{\alpha}>1$ and $\lambda_{2}=$ $\frac{\gamma \delta-\epsilon+\alpha \epsilon}{\gamma}$.

(iii) The Jacobian matrix of the linearized system of (1.1) about the fixed point $\left(0, \frac{-1+\delta}{\eta}\right)$ is given by

$$
F_{J}\left(0, \frac{-1+\delta}{\eta}\right)=\left[\begin{array}{cc}
\frac{\beta-\beta \delta+\alpha \eta}{\eta} & 0 \\
\frac{(-1+\delta) \epsilon}{\delta \eta} & \frac{1}{\delta}
\end{array}\right] .
$$


The eigenvalues of the Jacobian matrix $J_{F}\left(0, \frac{-1+\delta}{\eta}\right)$ about $\left(0, \frac{-1+\delta}{\eta}\right)$ are $\lambda_{1}=\frac{1}{\delta}>1$ and $\lambda_{2}=$ $\frac{\beta-\beta \delta+\alpha \eta}{\eta}$.

Theorem 3.2 The following statements are true.

(i) If $\alpha>1, \delta<1$, and $\epsilon<\frac{\gamma-\gamma \delta}{\alpha-1}$, then the equilibrium point $Q=\left(\frac{-1+\alpha}{\gamma}, 0\right)$ is locally asymptotically stable.

(ii) If $\delta>1$ and $\alpha<1$, then the equilibrium point $R=\left(0, \frac{-1+\delta}{\eta}\right)$ is locally asymptotically stable.

Theorem 3.3 Assume that $\alpha>1, \delta>1$, and $\eta>\frac{-\beta+\beta \delta}{-1+\alpha}$, then the unique equilibrium point $P=\left(\frac{\beta-\beta \delta+(-1+\alpha) \eta}{\beta \epsilon+\gamma \eta}, \frac{\gamma(-1+\delta)+(-1+\alpha) \epsilon}{\beta \epsilon+\gamma \eta}\right)$ is locally asymptotically stable if

$$
\Omega<(\beta(\gamma-\gamma \delta+\epsilon)+\alpha \gamma \eta)^{2}(\gamma \delta \eta+\epsilon(\beta+(-1+\alpha) \eta))
$$

where

$$
\begin{aligned}
\Omega= & \beta^{3} \epsilon\left(\gamma^{2} \delta^{3}+\alpha \gamma \delta^{2} \epsilon+(5 \alpha+\delta) \epsilon^{2}\right) \\
& +\beta^{2}\left(\gamma^{3} \delta^{2}+\gamma(1+\alpha(5+2 \alpha+7 \delta)) \epsilon^{2}+3 \alpha^{2} \epsilon^{3}\right) \eta \\
& +\beta \gamma\left(\gamma^{2}\left(2 \alpha+\delta^{2}\right)+\alpha \gamma(7+(3+\alpha) \delta) \epsilon+\left(1+\alpha^{2}+\alpha^{3}\right) \epsilon^{2}\right) \eta^{2} \\
& +\alpha \gamma^{2}(\gamma(1+\alpha+\delta)+\alpha \epsilon) \eta^{3} .
\end{aligned}
$$

Proof Assume that $\alpha>1, \delta>1$, and $\eta>\frac{-\beta+\beta \delta}{-1+\alpha}$. Let $L=\beta-\beta \delta+(-1+\alpha) \eta>0$. Then a characteristic polynomial of the Jacobian matrix $F_{J}(P)$ about the unique equilibrium point $P=\left(\frac{L}{\beta \epsilon+\gamma \eta}, \frac{\gamma(-1+\delta)+(-1+\alpha) \epsilon}{\beta \epsilon+\gamma \eta}\right)$ is given by

$$
\Upsilon(\lambda)=\lambda^{2}-(A-B+C) \lambda+D-E+F-G+H
$$

where

$$
\begin{aligned}
& A=\frac{\alpha}{\left(1+\frac{L \gamma}{\beta \epsilon+\gamma \eta}\right)^{2}}, \\
& B=\frac{\beta(\gamma(-1+\delta)+(-1+\alpha) \epsilon)(\beta \epsilon+\gamma \eta)}{(\beta \epsilon+\gamma(L+\eta))^{2}}, \\
& C=\frac{(\beta \epsilon+\gamma \eta)(\beta \delta \epsilon+\gamma \delta \eta+L \epsilon \lambda)}{(\gamma \delta \eta+\epsilon(\beta+(-1+\alpha) \eta))^{2}}, \\
& D=\frac{\alpha \delta(\beta \epsilon+\gamma \eta)^{4}}{(\beta \epsilon+\gamma(L+\eta))^{2}(\gamma \delta \eta+\epsilon(\beta+(-1+\alpha) \eta))^{2}}, \\
& E=\frac{\beta \delta(\gamma(-1+\delta)+(-1+\alpha) \epsilon)(\beta \epsilon+\gamma \eta)^{3}}{(\beta \epsilon+\gamma(L+\eta))^{2}(\gamma \delta \eta+\epsilon(\beta+(-1+\alpha) \eta))^{2}}, \\
& F=\frac{L \alpha \epsilon(\beta \epsilon+\gamma \eta)^{3}}{(\beta \epsilon+\gamma(L+\eta))^{2}(\gamma \delta \eta+\epsilon(\beta+(-1+\alpha) \eta))^{2}}, \\
& G=\frac{L \beta \epsilon(\gamma(-1+\delta)+(-1+\alpha) \epsilon)(\beta \epsilon+\gamma \eta)^{2}}{(\beta \epsilon+\gamma(L+\eta))^{2}(\gamma \delta \eta+\epsilon(\beta+(-1+\alpha) \eta))^{2}},
\end{aligned}
$$


and

$$
H=\frac{(\beta \epsilon+\gamma \eta)(L \epsilon+\beta \delta \epsilon+\gamma \delta \eta)}{(\gamma \delta \eta+\epsilon(\beta+(-1+\alpha) \eta))^{2}} .
$$

Let

$$
S(\lambda)=\lambda^{2}, \quad T(\lambda)=(A-B+C) \lambda-D+E-F+G-H .
$$

Assume that $\Omega<(\beta(\gamma-\gamma \delta+\epsilon)+\alpha \gamma \eta)^{2}(\gamma \delta \eta+\epsilon(\beta+(-1+\alpha) \eta))$. Then one has

$$
\begin{aligned}
|T(\lambda)| & \leq A+B+C+D+E+F+G+H \\
& <\frac{\Omega}{(\beta(\gamma-\gamma \delta+\epsilon)+\alpha \gamma \eta)^{2}(\gamma \delta \eta+\epsilon(\beta+(-1+\alpha) \eta))}<1 .
\end{aligned}
$$

Then, by Rouche's theorem, $S(\lambda)$ and $S(\lambda)-T(\lambda)$ have the same number of zeroes in an open unit disk $|\lambda|<1$. Hence, the unique positive equilibrium point $P$ is locally asymptotically stable.

\subsection{Global character}

Theorem 3.4 Let $I=[a, b]$ and $J=[c, d]$ be real intervals, and let $f: I \times J \rightarrow I$ and $g$ : $I \times J \rightarrow J$ be continuous functions. Consider the system (2.1) with initial conditions $\left(x_{0}, y_{0}\right) \in$ $I \times J$. Suppose that the following statements are true.

(i) $f(x, y)$ is non-decreasing in $x$ and non-increasing in $y$.

(ii) $g(x, y)$ is non-decreasing in both arguments.

(iii) If $\left(m_{1}, M_{1}, m_{2}, M_{2}\right) \in I^{2} \times J^{2}$ is a solution of the system

$$
\begin{array}{ll}
m_{1}=f\left(m_{1}, M_{2}\right), & M_{1}=f\left(M_{1}, m_{2}\right), \\
m_{2}=g\left(m_{1}, m_{2}\right), & M_{2}=g\left(M_{1}, M_{2}\right)
\end{array}
$$

such that $m_{1}=M_{1}$ and $m_{2}=M_{2}$, then there exists exactly one equilibrium point $(\bar{x}, \bar{y})$ of the system $(2.1)$ such that $\lim _{n \rightarrow \infty}\left(x_{n}, y_{n}\right)=(\bar{x}, \bar{y})$.

Proof According to the Brouwer fixed point theorem, the function $F: I \times J \rightarrow I \times J$ defined by $F(x, y)=F(f(x, y), g(x, y))$ has a fixed point $(\bar{x}, \bar{y})$, which is a fixed point of the system (2.1).

Assume that $m_{1}^{0}=a, M_{1}^{0}=b, m_{2}^{0}=c, M_{2}^{0}=d$ such that

$$
m_{1}^{i+1}=f\left(m_{1}^{i}, M_{2}^{i}\right), \quad M_{1}^{i+1}=f\left(M_{1}^{i}, m_{2}^{i}\right),
$$

and

$$
m_{2}^{i+1}=g\left(m_{1}^{i}, m_{2}^{i}\right), \quad M_{2}^{i+1}=g\left(M_{1}^{i}, M_{2}^{i}\right)
$$

Then

$$
m_{1}^{0}=a \leq f\left(m_{1}^{0}, M_{2}^{0}\right) \leq f\left(M_{1}^{0}, m_{2}^{0}\right) \leq b=M_{1}^{0},
$$


and

$$
m_{2}^{0}=c \leq g\left(m_{1}^{0}, m_{2}^{0}\right) \leq g\left(M_{1}^{0}, M_{2}^{0}\right) \leq d=M_{2}^{0} .
$$

Moreover, one has

$$
m_{1}^{0} \leq m_{1}^{1} \leq M_{1}^{1} \leq M_{1}^{0}
$$

and

$$
m_{2}^{0} \leq m_{2}^{1} \leq M_{2}^{1} \leq M_{2}^{0}
$$

We similarly have

$$
m_{1}^{1}=f\left(m_{1}^{0}, M_{2}^{0}\right) \leq f\left(m_{1}^{1}, M_{2}^{1}\right) \leq f\left(M_{1}^{1}, m_{2}^{1}\right) \leq f\left(M_{1}^{0}, m_{2}^{0}\right) \leq M_{1}^{1}
$$

and

$$
m_{2}^{1}=g\left(m_{1}^{0}, m_{2}^{0}\right) \leq g\left(m_{1}^{1}, m_{2}^{1}\right) \leq g\left(M_{1}^{1}, M_{2}^{1}\right) \leq g\left(M_{1}^{0}, M_{2}^{0}\right) \leq M_{2}^{1} .
$$

Now observe that for each $i \geq 0$,

$$
a=m_{1}^{0} \leq m_{1}^{1} \leq \cdots \leq m_{1}^{i} \leq M_{1}^{i} \leq M_{1}^{i-1} \leq \cdots \leq M_{1}^{0}=b
$$

and

$$
c=m_{2}^{0} \leq m_{2}^{1} \leq \cdots \leq m_{2}^{i} \leq M_{2}^{i} \leq M_{2}^{i-1} \leq \cdots \leq M_{2}^{0}=d
$$

Hence, $m_{1}^{i} \leq x_{n} \leq M_{1}^{i}$, and $m_{2}^{i} \leq y_{n} \leq M_{2}^{i}$ for $n \geq 2 i+1$. Let $m_{1}=\lim _{n \rightarrow \infty} m_{1}^{i}, M_{1}=$ $\lim _{n \rightarrow \infty} M_{1}^{i}, m_{2}=\lim _{n \rightarrow \infty} m_{2}^{i}$, and $M_{2}=\lim _{n \rightarrow \infty} M_{2}^{i}$. Then $a \leq m_{1} \leq M_{1} \leq b$ and $c \leq m_{2} \leq$ $M_{2} \leq d$. By the continuity of $f$ and $g$, one has

$$
\begin{array}{ll}
m_{1}=f\left(m_{1}, M_{2}\right), & M_{1}=f\left(M_{1}, m_{2}\right), \\
m_{2}=g\left(m_{1}, m_{2}\right), & M_{2}=g\left(M_{1}, M_{2}\right) .
\end{array}
$$

Hence, $m_{1}=M_{1}, m_{2}=M_{2}$.

Theorem 3.5 Assume that $\eta \gamma-\beta \epsilon \neq 0$, then the unique positive equilibrium point $P$ of the system (1.1) is a global attractor.

Proof Let $f(x, y)=\frac{\alpha x-\beta x y}{1+\gamma x}$ and $g(x, y)=\frac{\delta y+\epsilon x y}{1+\eta y}$. Then it is easy to see that $f(x, y)$ is nondecreasing in $x$ and non-increasing in $y$. Moreover, $g(x, y)$ is non-decreasing in both $x$ and $y$. Let $\left(m_{1}, M_{1}, m_{2}, M_{2}\right)$ be a positive solution of the system

$$
\begin{array}{ll}
m_{1}=f\left(m_{1}, M_{2}\right), & M_{1}=f\left(M_{1}, m_{2}\right), \\
m_{2}=g\left(m_{1}, m_{2}\right), & M_{2}=g\left(M_{1}, M_{2}\right) .
\end{array}
$$


Then one has

$$
m_{1}=\frac{\alpha m_{1}-\beta m_{1} M_{2}}{1+\gamma m_{1}}, \quad M_{1}=\frac{\alpha M_{1}-\beta M_{1} m_{2}}{1+\gamma M_{1}},
$$

and

$$
m_{2}=\frac{\delta m_{2}+\epsilon m_{1} m_{2}}{1+\eta m_{2}}, \quad M_{2}=\frac{\delta M_{2}+\epsilon M_{1} M_{2}}{1+\eta M_{2}} .
$$

From (3.1), one has

$$
1+\gamma m_{1}=\alpha-\beta M_{2}, \quad 1+\gamma M_{1}=\alpha-\beta m_{2} .
$$

On subtraction, (3.3) implies that

$$
\gamma\left(m_{1}-M_{1}\right)=\beta\left(m_{2}-M_{2}\right) .
$$

Similarly, from (3.2), one has

$$
1+\eta m_{2}=\delta+\epsilon m_{1}, \quad 1+\eta M_{2}=\delta+\epsilon M_{1} .
$$

On subtraction, (3.5) implies that

$$
\eta\left(m_{2}-M_{2}\right)=\epsilon\left(m_{1}-M_{1}\right) .
$$

Comparing (3.4) and (3.6), one has

$$
(\eta \gamma-\beta \epsilon)\left(m_{1}-M_{1}\right)=0
$$

Then one has $m_{1}=M_{1}$ and $m_{2}=M_{2}$. Hence, from Theorem 3.4 the equilibrium point $\left(\frac{\beta-\beta \delta+(-1+\alpha) \eta}{\beta \epsilon+\gamma \eta}, \frac{\gamma(-1+\delta)+(-1+\alpha) \epsilon}{\beta \epsilon+\gamma \eta}\right)$ of the system (1.1) is a global attractor.

Theorem 3.6 Assume that $\alpha>1, \delta>1$, and $\eta \gamma-\beta \epsilon \neq 0$. Then the unique positive equilibrium point $(\bar{x}, \bar{y})=\left(\frac{\beta-\beta \delta+(-1+\alpha) \eta}{\beta \epsilon+\gamma \eta}, \frac{\gamma(-1+\delta)+(-1+\alpha) \epsilon}{\beta \epsilon+\gamma \eta}\right)$ is globally asymptotically stable.

Proof The proof follows from Theorem 3.3 and Theorem 3.5.

\subsection{Rate of convergence}

In this section we determine the rate of convergence of a solution that converges to the unique positive equilibrium point of the system (1.1)

The following result gives the rate of convergence of solutions of a system of difference equations:

$$
X_{n+1}=(A+B(n)) X_{n},
$$

where $X_{n}$ is an $m$-dimensional vector, $A \in C^{m \times m}$ is a constant matrix, and $B: \mathbb{Z}^{+} \rightarrow C^{m \times m}$ is a matrix function satisfying

$$
\|B(n)\| \rightarrow 0
$$


as $n \rightarrow \infty$, where $\|\cdot\|$ denotes any matrix norm which is associated with the vector norm

$$
\|(x, y)\|=\sqrt{x^{2}+y^{2}}
$$

Proposition 3.7 (Perron's theorem [28]) Suppose that condition (3.8) holds. If $X_{n}$ is a solution of (3.7), then either $X_{n}=0$ for all large $n$ or

$$
\rho=\lim _{n \rightarrow \infty}\left(\left\|X_{n}\right\|\right)^{1 / n}
$$

exists and is equal to the modulus of one of the eigenvalues of matrix A.

Proposition 3.8 [28] Suppose that condition (3.8) holds. If $X_{n}$ is a solution of (3.7), then either $X_{n}=0$ for all large $n$ or

$$
\rho=\lim _{n \rightarrow \infty} \frac{\left\|X_{n+1}\right\|}{\left\|X_{n}\right\|}
$$

exists and is equal to the modulus of one of the eigenvalues of matrix $A$.

Let $\left\{\left(x_{n}, y_{n}\right)\right\}$ be any solution of the system (1.1) such that $\lim _{n \rightarrow \infty} x_{n}=\bar{x}$, and $\lim _{n \rightarrow \infty} y_{n}=$ $\bar{y}$, where $(\bar{x}, \bar{y})=\left(\frac{\beta-\beta \delta+(-1+\alpha) \eta}{\beta \epsilon+\gamma \eta}, \frac{\gamma(-1+\delta)+(-1+\alpha) \epsilon}{\beta \epsilon+\gamma \eta}\right)$. To find the error terms, one has from the system (1.1)

$$
\begin{aligned}
x_{n+1}-\bar{x} & =\frac{\alpha x_{n}-\beta x_{n} y_{n}}{1+\gamma x_{n}}-\frac{\alpha \bar{x}-\beta \bar{x} \bar{y}}{1+\gamma \bar{x}} \\
& =\frac{\left(\alpha-\beta y_{n}\right)}{\left(1+\gamma x_{n}\right)(1+\gamma \bar{x})}\left(x_{n}-\bar{x}\right)-\frac{\beta \bar{x}}{1+\gamma \bar{x}}\left(y_{n}-\bar{y}\right),
\end{aligned}
$$

and

$$
\begin{aligned}
y_{n+1}-\bar{y} & =\frac{\delta y_{n}+\epsilon x_{n} y_{n}}{1+\eta y_{n}}-\frac{\delta \bar{y}+\epsilon \bar{x} \bar{y}}{1+\eta \bar{y}} \\
& =\frac{\epsilon \bar{y}}{1+\eta \bar{y}}\left(x_{n}-\bar{x}\right)+\frac{\delta+\epsilon x_{n}}{\left(1+\eta y_{n}\right)(1+\epsilon \bar{y})}\left(y_{n}-\bar{x}\right) .
\end{aligned}
$$

Let $e_{n}^{1}=x_{n}-\bar{x}$ and $e_{n}^{2}=y_{n}-\bar{y}$, then one has

$$
e_{n+1}^{1}=a_{n} e_{n}^{1}+b_{n} e_{n}^{2}
$$

and

$$
e_{n+1}^{2}=c_{n} e_{n}^{1}+d_{n} e_{n}^{2}
$$

where

$$
\begin{aligned}
& a_{n}=\frac{\left(\alpha-\beta y_{n}\right)}{\left(1+\gamma x_{n}\right)(1+\gamma \bar{x})}, \quad b_{n}=-\frac{\beta \bar{x}}{1+\gamma \bar{x}}, \\
& c_{n}=\frac{\epsilon \bar{y}}{1+\eta \bar{y}}, \quad d_{n}=\frac{\delta+\epsilon x_{n}}{\left(1+\eta y_{n}\right)(1+\epsilon \bar{y})} .
\end{aligned}
$$


Moreover,

$$
\begin{aligned}
& \lim _{n \rightarrow \infty} a_{n}=\frac{\alpha-\bar{y} \beta}{(1+\bar{x} \gamma)^{2}}, \quad \lim _{n \rightarrow \infty} b_{n}=-\frac{\bar{x} \beta}{1+\bar{x} \gamma}, \\
& \lim _{n \rightarrow \infty} c_{n}=\frac{\bar{y} \epsilon}{1+\bar{y} \eta}, \quad \lim _{n \rightarrow \infty} d_{n}=\frac{\delta+\bar{x} \epsilon}{(1+\bar{y} \eta)^{2}} .
\end{aligned}
$$

Now the limiting system of error terms can be written as

$$
\left[\begin{array}{l}
e_{n+1}^{1} \\
e_{n+1}^{2}
\end{array}\right]=\left[\begin{array}{cc}
\frac{\alpha-\bar{y} \beta}{(1+\bar{x} \gamma)^{2}} & -\frac{\bar{x} \beta}{1+\bar{x} \gamma} \\
\overline{\bar{y} \epsilon} & \frac{\delta+\bar{x} \epsilon}{1+\bar{y} \eta}
\end{array}\right]\left[\begin{array}{l}
e_{n}^{1} \\
e_{n}^{2}
\end{array}\right]
$$

which is similar to the linearized system of (1.1) about the equilibrium point $(\bar{x}, \bar{y})$.

Using Proposition 3.7, one has following result.

Theorem 3.9 Assume that $\left\{\left(x_{n}, y_{n}\right)\right\}$ is a positive solution of the system (1.1) such that $\lim _{n \rightarrow \infty} x_{n}=\bar{x}$ and $\lim _{n \rightarrow \infty} y_{n}=\bar{y}$, where

$$
(\bar{x}, \bar{y})=\left(\frac{\beta-\beta \delta+(-1+\alpha) \eta}{\beta \epsilon+\gamma \eta}, \frac{\gamma(-1+\delta)+(-1+\alpha) \epsilon}{\beta \epsilon+\gamma \eta}\right) .
$$

Then the error vector $e_{n}=\left(\begin{array}{c}e_{n}^{1} \\ e_{n}^{2}\end{array}\right)$ of every solution of (1.1) satisfies both of the following asymptotic relations:

$$
\lim _{n \rightarrow \infty}\left(\left\|e_{n}\right\|\right)^{\frac{1}{n}}=\left|\lambda_{1,2} F_{J}(\bar{x}, \bar{y})\right|, \quad \lim _{n \rightarrow \infty} \frac{\left\|e_{n+1}\right\|}{\left\|e_{n}\right\|}=\left|\lambda_{1,2} F_{J}(\bar{x}, \bar{y})\right|
$$

where $\lambda_{1,2} F_{J}(\bar{x}, \bar{y})$ are the characteristic roots of the Jacobian matrix $F_{J}(\bar{x}, \bar{y})$.

\section{Examples}

In this section, we consider some numerical examples which show that under a suitable choice of parameters $\alpha, \beta, \gamma, \delta, \epsilon, \eta$, the unique positive equilibrium point $\left(\frac{\beta-\beta \delta+(-1+\alpha) \eta}{\beta \epsilon+\gamma \eta}\right.$, $\left.\frac{\gamma(-1+\delta)+(-1+\alpha) \epsilon}{\beta \epsilon+\gamma \eta}\right)$ of the system (1.1) is globally asymptotically stable.

Example Let $\alpha=1.001, \beta=0.03, \gamma=0.6, \delta=1.002, \epsilon=1.7, \eta=0.9$. Then the system (1.1) can be written as

$$
x_{n+1}=\frac{1.001 x_{n}-0.03 x_{n} y_{n}}{1+0.6 x_{n}}, \quad y_{n+1}=\frac{1.002 y_{n}+1.7 x_{n} y_{n}}{1+0.9 y_{n}}
$$

with initial conditions $x_{0}=0.0002, y_{0}=0.0006$.

In this case, the unique positive equilibrium point $P$ of the system (4.1) is given by

$$
\left(\frac{\beta-\beta \delta+(-1+\alpha) \eta}{\beta \epsilon+\gamma \eta}, \frac{\gamma(-1+\delta)+(-1+\alpha) \epsilon}{\beta \epsilon+\gamma \eta}\right)=(0.00142132,0.00490694) .
$$

Moreover, the plot is shown in Figure 1. 

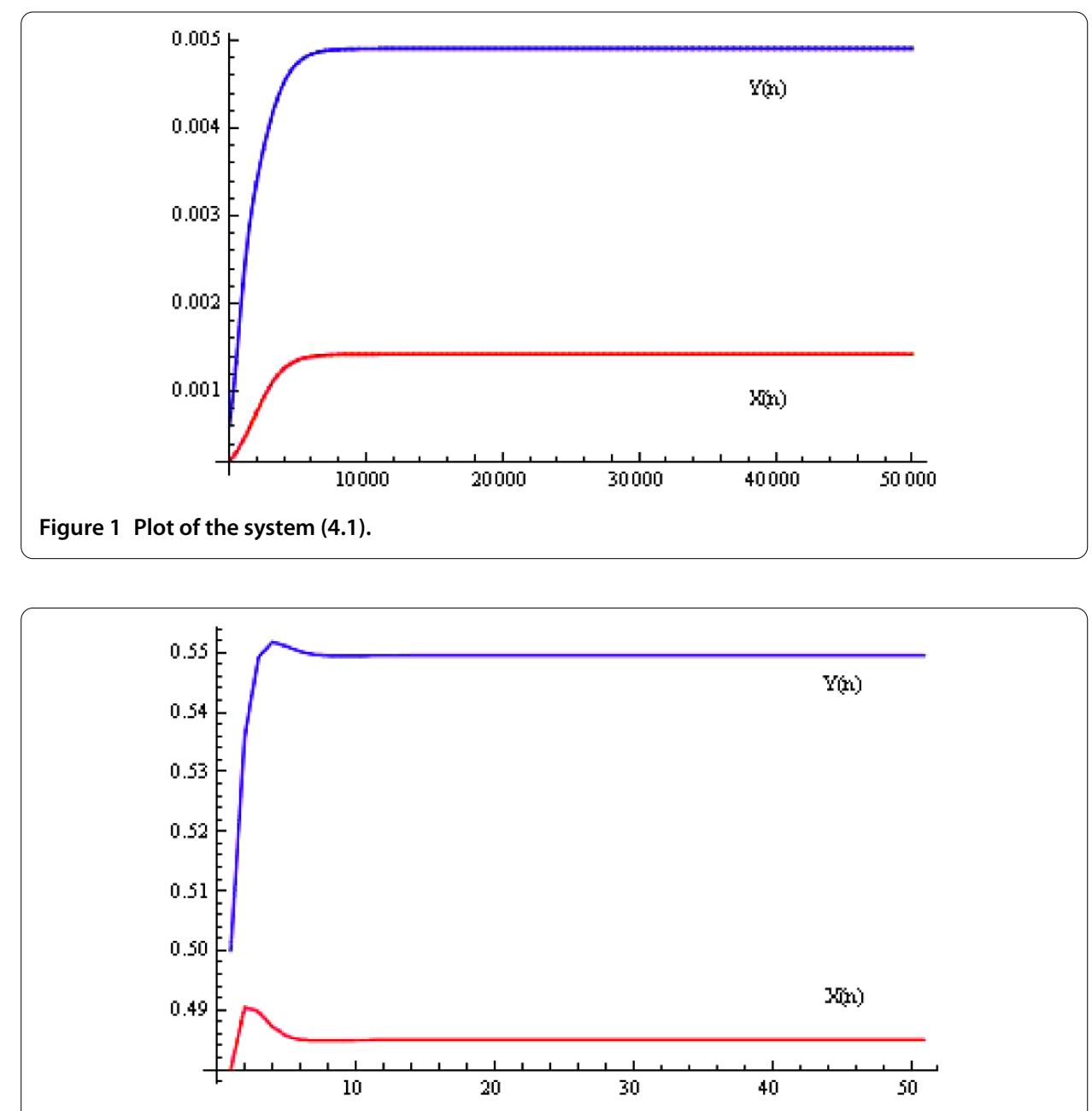

Figure 2 Plot of the system (4.2).

Example Let $\alpha=2.5, \beta=0.7, \gamma=2.3, \delta=2.7, \epsilon=4.2, \eta=6.8$. Then the system (1.1) can be written as

$$
x_{n+1}=\frac{2.5 x_{n}-0.7 x_{n} y_{n}}{1+2.3 x_{n}}, \quad y_{n+1}=\frac{2.7 y_{n}+4.2 x_{n} y_{n}}{1+6.8 y_{n}},
$$

with initial conditions $x_{0}=0.84, y_{0}=0.5$.

In this case, the unique equilibrium point $P$ of the system (4.2) is given by

$$
\left(\frac{\beta-\beta \delta+(-1+\alpha) \eta}{\beta \epsilon+\gamma \eta}, \frac{\gamma(-1+\delta)+(-1+\alpha) \epsilon}{\beta \epsilon+\gamma \eta}\right)=(0.48493,0.549516) .
$$

Moreover, the plot is shown in Figure 2.

Example Let $\alpha=22, \beta=1.7, \gamma=20.5, \delta=6, \epsilon=0.2, \eta=2.8$. Then the system (1.1) can be written as

$$
x_{n+1}=\frac{2.5 x_{n}-1.7 x_{n} y_{n}}{1+20.5 x_{n}}, \quad y_{n+1}=\frac{6 y_{n}+0.2 x_{n} y_{n}}{1+2.8 y_{n}},
$$

with initial conditions $x_{0}=0.7, y_{0}=0.8$. 


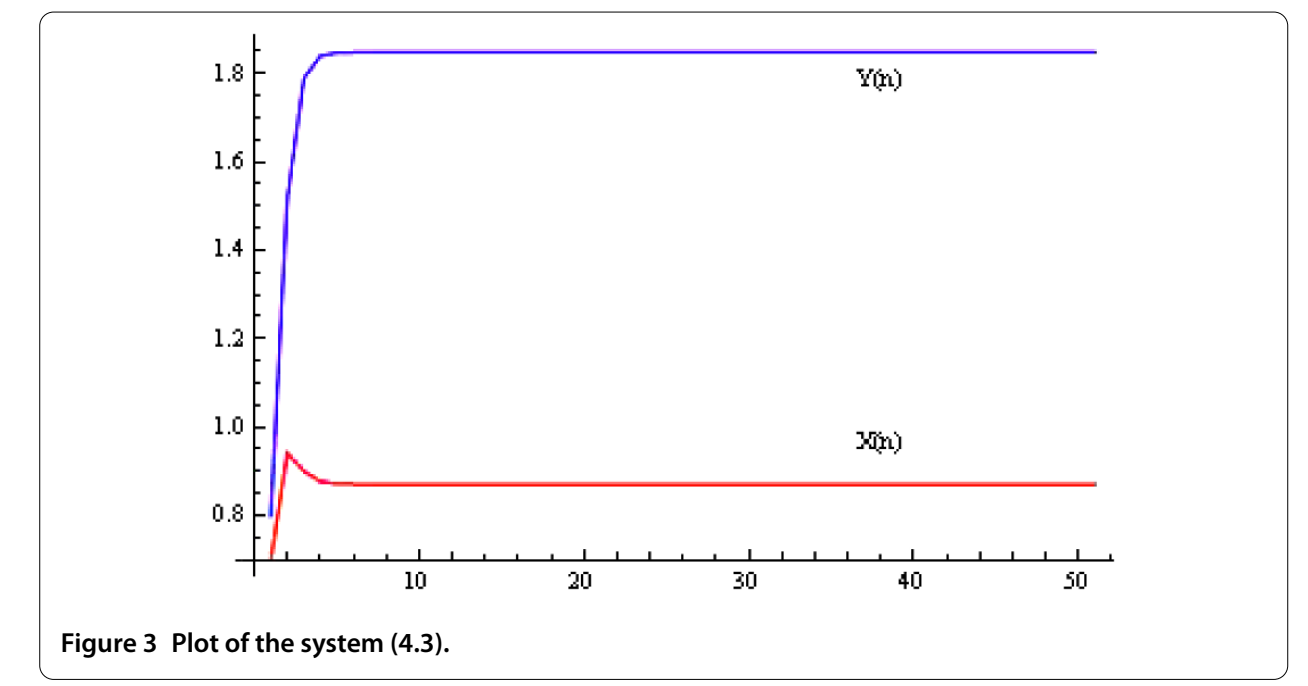

In this case, the unique equilibrium point $P$ of the system (4.3) is given by

$$
\left(\frac{\beta-\beta \delta+(-1+\alpha) \eta}{\beta \epsilon+\gamma \eta}, \frac{\gamma(-1+\delta)+(-1+\alpha) \epsilon}{\beta \epsilon+\gamma \eta}\right)=(0.871147,1.84794)
$$

Moreover, the plot is shown in Figure 3.

Example Let $\alpha=170, \beta=11, \gamma=2.7, \delta=50, \epsilon=1.7, \eta=7$. Then the system (1.1) can be written as

$$
x_{n+1}=\frac{170 x_{n}-11 x_{n} y_{n}}{1+2.7 x_{n}}, \quad y_{n+1}=\frac{50 y_{n}+1.7 x_{n} y_{n}}{1+7 y_{n}},
$$

with initial conditions $x_{0}=7, y_{0}=5$.

In this case, the unique positive equilibrium point $P$ of the system (4.4) is given by

$$
\left(\frac{\beta-\beta \delta+(-1+\alpha) \eta}{\beta \epsilon+\gamma \eta}, \frac{\gamma(-1+\delta)+(-1+\alpha) \epsilon}{\beta \epsilon+\gamma \eta}\right)=(17.1276,11.1597)
$$

Moreover, the plot of the system (4.4) is shown in Figure 4. An attractor of the system is shown in Figure 5.

\section{Conclusions}

This work is related to the qualitative behavior of a discrete-time Lotka-Volterra model. The continuous form of this model is given by

$$
\frac{d x}{d t}=a x-b x^{2}-c x y, \quad \frac{d y}{d t}=m x y+n y-p y^{2},
$$

where $a, b, c, m, n, p$ are positive constants. Moreover, the discrete form (1.1) of the continuous model is obtained by using some nonstandard difference scheme such that the equilibrium points in both cases are conserved. We proved that the system (1.1) has four equilibrium points, which are locally asymptotically stable under certain conditions. The 

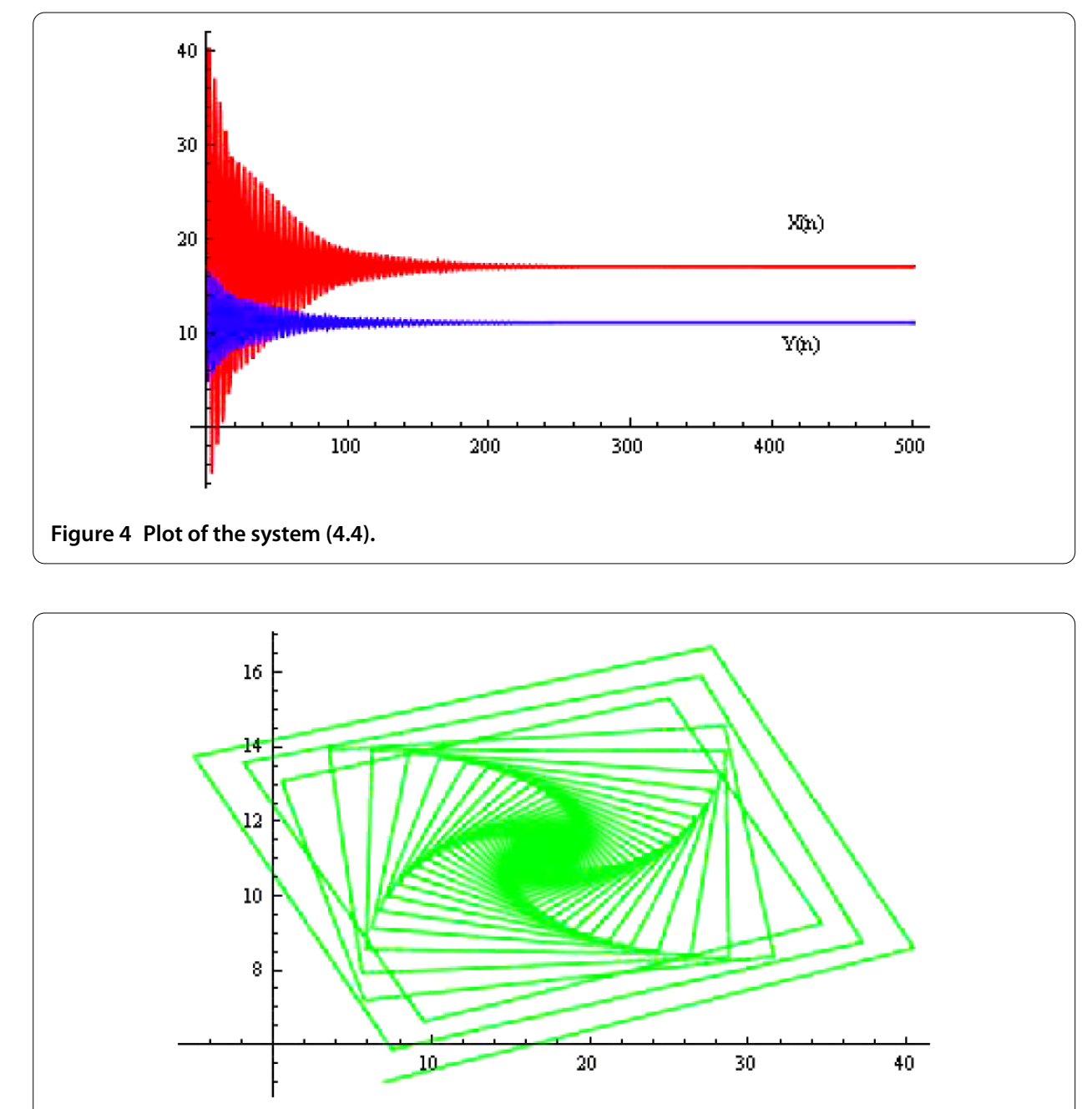

Figure 5 An attractor of the system (4.4).

main contribution in this paper is to prove that the unique positive equilibrium point

$$
P=\left(\frac{\beta-\beta \delta+(-1+\alpha) \eta}{\beta \epsilon+\gamma \eta}, \frac{\gamma(-1+\delta)+(-1+\alpha) \epsilon}{\beta \epsilon+\gamma \eta}\right)
$$

of the system (1.1) is globally asymptotically stable. Furthermore, we have investigated the rate of convergence of the solution that converges to the unique positive equilibrium point of the system (1.1). Some numerical examples are provided to support our theoretical results. These examples are experimental verifications of theoretical discussions.

\section{Competing interests}

The author declares that he has no competing interests.

\section{Author's contributions}

The author carried out the proof of the main results and approved the final manuscript.

\section{Acknowledgements}

The author would like to thank the main editor and anonymous referees for their valuable comments and suggestions leading to improvement of this paper. This work was supported by the Higher Education Commission of Pakistan. 


\section{References}

1. Ahmad, S: On the nonautonomous Lotka-Volterra competition equation. Proc. Am. Math. Soc. 117, $199-204$ (1993)

2. Tang, X, Zou, X: On positive periodic solutions of Lotka-Volterra competition systems with deviating arguments. Proc. Am. Math. Soc. 134, 2967-2974 (2006)

3. Zhou, Z, Zou, X: Stable periodic solutions in a discrete periodic logistic equation. Appl. Math. Lett. 16(2), 165-171 (2003)

4. Liu, X: A note on the existence of periodic solution in discrete predator-prey models. Appl. Math. Model. 34, $2477-2483(2010)$

5. Krebs, W: A general predator-prey model. Math. Comput. Model. Dyn. Syst. 9, 387-401 (2003)

6. Allen, LJS: An Introduction to Mathematical Biology. Prentice Hall, New York (2007)

7. Brauer, F, Castillo-Chavez, C: Mathematical Models in Population Biology and Epidemiology. Springer, Berlin (2000)

8. Edelstein-Keshet, L: Mathematical Models in Biology. McGraw-Hill, New York (1988)

9. Kulenović, MRS, Ladas, G: Dynamics of Second Order Rational Difference Equations. Chapman \& Hall/CRC Press, Boca Raton (2002)

10. Camouzis, E, Ladas, G: Dynamics of Third-Order Rational Difference Equations with Open Problems and Conjectures. Chapman \& Hall/CRC Press, Boca Raton (2007)

11. Elaydi, S: An Introduction to Difference Equations, 3rd edn. Springer, New York (2005)

12. Agarwal, RP, Wong, P: Advanced Topics in Difference Equations. Kluwer Academic, Dordrecht (1997)

13. Agarwal, RP: Difference Equations and Inequalities, 1st edn. Dekker, New York (1992) (2nd edn. (2000))

14. Grove, EA, Ladas, G: Periodicities in Nonlinear Difference Equations. Chapman \& Hall/CRC Press, Boca Raton (2004)

15. Aloqeili, M: Dynamics of a rational difference equation. Appl. Math. Comput. 176(2), 768-776 (2006)

16. Cinar, C: On the positive solutions of the difference equation system $x_{n+1}=\frac{1}{y_{n}} ; y_{n+1}=\frac{y_{n}}{x_{n-1} y_{n-1}}$. Appl. Math. Comput. 158, 303-305 (2004)

17. Stević, S: On some solvable systems of difference equations. Appl. Math. Comput. 218, 5010-5018 (2012)

18. Bajo, I, Liz, E: Global behaviour of a second-order nonlinear difference equation. J. Differ. Equ. Appl. 17(10), 1471-1486 (2011)

19. Kalabuŝić, S, Kulenović, MRS, Pilav, E: Dynamics of a two-dimensional system of rational difference equations of Leslie-Gower type. Adv. Differ. Equ. (2011). doi:10.1186/1687-1847-2011-29

20. Touafek, N, Elsayed, EM: On the solutions of systems of rational difference equations. Math. Comput. Model. 55 1987-1997 (2012)

21. Touafek, N, Elsayed, EM: On the periodicity of some systems of nonlinear difference equations. Bull. Math. Soc. Sci. Math. Roum. 2, 217-224 (2012)

22. Din, Q: On a system of rational difference equation. Demonstr. Math. (in press)

23. Din, Q: Global character of a rational difference equation. Thai J. Math. (in press)

24. Din, Q, Qureshi, MN, Khan, AQ: Dynamics of a fourth-order system of rational difference equations. Adv. Differ. Equ. (2012). doi:10.1186/1687-1847-2012-216

25. Zhang, Q, Yang, L, Liu, J: Dynamics of a system of rational third order difference equation. Adv. Differ. Equ. (2012). doi:10.1186/1687-1847-2012-136

26. Shojaei, M, Saadati, R, Adibi, H: Stability and periodic character of a rational third order difference equation. Chaos Solitons Fractals 39, 1203-1209 (2009)

27. Elsayed, EM: Behavior and expression of the solutions of some rational difference equations. J. Comput. Anal. Appl. 15(1), 73-81 (2013)

28. Pituk, M: More on Poincare's and Perron's theorems for difference equations. J. Differ. Equ. Appl. 8, 201-216 (2002)

doi:10.1186/1687-1847-2013-95

Cite this article as: Din: Dynamics of a discrete Lotka-Volterra model. Advances in Difference Equations 2013 2013:95.

\section{Submit your manuscript to a SpringerOpen ${ }^{\circ}$ journal and benefit from:}

- Convenient online submission

- Rigorous peer review

- Immediate publication on acceptance

- Open access: articles freely available online

- High visibility within the field

- Retaining the copyright to your article 